\title{
Clinical Characteristics of Patients Carrying the Q703K Variant of the NLRP3 Gene: A 10-year Multicentric National Study
}

\author{
Aldo Naselli, Federica Penco, Luca Cantarini, Antonella Insalaco, Mariolina Alessio, \\ Alberto Tommasini, Cristina Maggio, Laura Obici, Romina Gallizi, Marco Cimmino, \\ Sara Signa, Orso Maria Lucherini, Sonia Carta, Francesco Caroli, Alberto Martini, \\ Anna Rubartelli, Isabella Ceccherini, and Marco Gattorno
}

\begin{abstract}
Objective. The aim of our study was to analyze the clinical and functional effect of the p.Q703K (p. Q705K, c. $2107 \mathrm{C}>\mathrm{A}$ ) variant of the NLRP3 gene in a population of patients screened for suspected cryopyrin-associated periodic syndrome (CAPS).

Methods. Since 2002, 580 patients underwent molecular analysis for NLRP3. Data on clinical presentation, response to treatment, and longterm followup were collected using a uniform questionnaire. The pattern of cytokine secretion after lipopolysaccharide stimulation from isolated monocytes was analyzed in 3 patients carrying the p.Q703K variant and 1 patient with a chronic infantile neurologic, cutaneous, articular syndrome phenotype carrying both the p.M406I and p.Q703K, and compared with 7 patients with CAPS with sure pathogenic variants and 6 healthy controls .

Results. The p.Q703K variant was found in 57 screened patients with an overall allelic frequency of $5 \%$. The frequency in normal controls was $5.5 \%$. Clinical data at the moment of molecular analysis and at followup were available in 36 patients. Two patients displayed additional mutations of NLRP3. The mean followup was 2.5 years. Thirteen patients (39\%) had a final diagnosis different from the original suspicion of CAPS. The remaining 21 patients displayed a mild phenotype mainly characterized by recurrent episodes of urticarial rash and arthralgia. Only 8 patients were treated with antiinterleukin (IL)-1 treatment, with a complete response in 5 patients. The pattern of secretion of IL- $1 \beta$ and other cytokines (IL-6 and IL-1 receptor antagonist) in patients did not display the aberrancies observed in patients with CAPS and was similar to that observed in healthy controls.

Conclusion. The present study confirms the weak clinical and functional effect of the p.Q703K variant. (First Release April 1 2016; J Rheumatol 2016;43:1093-1100; doi:10.3899/jrheum.150962)
\end{abstract}

Key Indexing Terms:

NLRP3 INFLAMMASOME

CRYOPYRIN-ASSOCIATED PERIODIC SYNDROME

CRYOPYRIN

INTERLEUKIN-1 $\beta$

From the Unità Operativa (UO) Pediatria 2, IRCCS G. Gaslini; Clinica Reumatologica, Dipartimento di Medicina Interna, Università di Genova; Laboratorio di Biologia Cellulare, IRCCS Azienda Ospedaliera Universitaria (AOU) San Martino Istituto Scientifico Tumori (IST); Unità Operativa Complessa (UOC) Genetica Medica, IRCCS G. Gaslini; Università degli Studi di Genova, Genoa; Dipartimento di Reumatologia, Policlinico Le Scotte, Università di Siena, UO Reumatologia, Siena; Ospedale Bambino Gesù, Rome; Dipartimento di Pediatria, Ospedale Federico II, Naples; Dipartimento di Pediatria, IRCCS Burlo Garofalo, Trieste; Dipartimento di Scienze per la Promozione della Salute e Materno Infantile “G. D'Alessandro", Palermo; Centro per lo Studio e la Cura delle Amiloidosi Sistemiche Fondazione IRCCS Policlinico San Matteo, Pavia; Dipartimento Materno-Infantile, Ospedale Gaetano-Martino, Messina, Italy

A. Naselli*, MD, UO Pediatria 2, IRCCS G. Gaslini; F. Penco*, MD, UO Pediatria 2, IRCCS G. Gaslini; L. Cantarini, MD, Dipartimento di

Reumatologia, Policlinico Le Scotte, Università di Siena, UO

Reumatologia; A. Insalaco, MD, Ospedale Bambino Gesù; M. Alessio, MD, Dipartimento di Pediatria, Ospedale Federico II; A. Tommasini, MD, Dipartimento di Pediatria, IRCCS Burlo Garofalo; C. Maggio, MD,
Dipartimento di Scienze per la Promozione della Salute e Materno Infantile "G. D’Alessandro"; L. Obici, MD, Centro per lo Studio e la Cura delle Amiloidosi Sistemiche Fondazione IRCCS Policlinico San Matteo; R. Gallizi, MD, Dipartimento Materno-Infantile, Ospedale Gaetano-Martino; M. Cimmino, MD, Clinica Reumatologica, Dipartimento di Medicina Interna, Università di Genova; S. Signa, MD, UO Pediatria 2, IRCCS G. Gaslini; O.M. Lucherini, MD, Dipartimento di Reumatologia, Policlinico Le Scotte, Università di Siena, UO Reumatologia; S. Carta, MD, Laboratorio di Biologia Cellulare, IRCCS AOU San Martino IST; F. Caroli, MD, UOC Genetica Medica, IRCCS G. Gaslini; A. Martini, MD, UO Pediatria 2, IRCCS G. Gaslini, and Università degli Studi di Genova; A. Rubartelli, MD, Laboratorio di Biologia Cellulare, IRCCS AOU San Martino IST; I. Ceccherini, MD, UOC Genetica Medica, IRCCS G. Gaslini; M. Gattorno, MD, UO Pediatria 2, IRCCS G. Gaslini.* These authors equally contributed to the paper.

Address correspondence to Dr. M. Gattorno, UO Pediatria 2, IRCCS Giannina Gaslini Institute and University of Genoa, Largo G. Gaslini 5, 16147 Genoa, Italy.E-mail: marcogattorno@ospedale-gaslini.ge.it Accepted for publication February 4, 2016.

Personal non-commercial use only. The Journal of Rheumatology Copyright @ 2016 . All rights reserved. 
Cryopyrin-associated periodic syndromes (CAPS) are a group of autoinflammatory diseases associated to dominantly inherited or de novo mutations of the NLRP3 gene ${ }^{1}$. Representing the clinical spectrum of CAPS are familial cold autoinflammatory syndrome (FCAS), Muckle-Wells syndrome (MWS), and neonatal-onset multisystem inflammatory disease [NOMID; also known as chronic infantile neurologic, cutaneous, articular syndrome (CINCA syndrome) $]^{2,3,4,5,6}$.

To date, more than 170 different variants of NLRP3 have been identified (fmf.igh.cnrs.fr/Infevers), the large majority of which is localized in the exon 3 that encodes the oligomerization (NLR-binding) domain of cryopyrin ${ }^{7}$. Somatic mosaicisms have been reported in patients with a severe CAPS phenotype negative to germ-line mutations of $N L R P 3^{8,9}$. Pathogenic variants of $N L R P 3$ result in substantial upregulation of caspase 1-mediated cleavage of pro-interleukin (IL)- $1 \beta$ and secretion of active IL- $1 \beta^{10,11}$. For this reason, IL-1 blockade is extremely effective in these patients ${ }^{12,13,14}$. Even if a strict genotype-phenotype correlation cannot be ascertained, it is now clear that some variants found with high penetrance in large families are usually associated to a milder phenotype, whereas rare and de novo mutations are usually associated to the more severe CINCA phenotype ${ }^{3,4,15}$. On the other hand, other variants, such as V198M and I313V, are characterized by a low penetrance and their clinical effect is debated ${ }^{7,16}$. The p.Q703K variant (p. Q705K, c. 2107C >A) has been considered for many years as a clinically unremarkable polymorphism because of its presence in $5-11 \%$ of the general population (fmf.igh.cnrs.fr $)^{7,17}$. However, 2 subsequent studies from Sweden have shown that the p.Q703K variant displays a higher secretion of IL-1 $\beta$, thus suggesting a possible pathogenic involvement of this variant ${ }^{18,19}$. This hypothesis was supported by a preliminary clinical study performed in 7 adult patients $^{20}$.

The aims of our study were (1) to analyze the prevalence of p.Q703K mutation in pediatric and adult patients screened for NLPR3 in the context of a diagnostic workup for a suspected CAPS and to compare it to its prevalence in the normal population, (2) to describe the clinical findings at presentation, the disease outcome, and response to treatment in patients carrying the p.Q703K NLRP3 variant, and (3) to analyze the pattern of IL-1 $\beta$ secretion in this subgroup of patients.

\section{MATERIALS AND METHODS}

Patients' selection. From March 2002 to January 2013, molecular analysis of the NLRP 3 was performed in 580 patients for a clinical suspicion of CAPS and other periodic fevers by 2 national referral centers ( 1 pediatric, 1 adult) collecting samples from Italian centers (local hospitals, secondary and tertiary referral centers) distributed in all regions of the country. Subjects who displayed features possibly associated to autoinflammatory diseases other than CAPS or presenting an unspecific phenotype underwent additional screening for the following genes associated to monogenic periodic fevers: $M E F V$ [associated with familial Mediterranean fever (FMF)], TNFRSF1A [associated with tumor necrosis factor (TNF) receptor-associated periodic syndrome], $M V K$ (associated with mevalonate kinase deficiency), and NLRP12 (FCAS 2). All patients carrying the p.Q703K (p. Q705K, c. $2107 \mathrm{C}>\mathrm{A})$ NLRP3 variant were enrolled in our study. Detailed information concerning the family history, personal history, and clinical manifestations at disease presentation were collected at the time of genetic analysis with a uniform questionnaire.

To collect information on the followup of the patients, a second questionnaire was sent to all participating centers on March 2013. Data were collected on modification of the clinical picture during the followup, including the possible appearance of complications such as hearing loss, eye manifestations, or amyloidosis. Data were also evaluated on the association between the clinical manifestations and the elevation of acute-phase reactants and on response to different therapeutic strategies. Centers were also asked to indicate whether a possible alternative diagnosis was made during the followup period subsequent to the genetic analysis. The study was approved by the ethics board of the G. Gaslini Institute, Genoa, Italy. Local ethical boards also approved the study in each center.

Molecular analysis of NLRP3. The exon $3(1.7 \mathrm{~Kb})$ of the gene was amplified by PCR and the 5 overlapping amplification products were sequenced by using the BigDye Terminator Cycle Sequencing Ready reaction Kit 3.0 and loading the reactions into an automated DNA sequencer ${ }^{21}$.

Cell preparation and culture. These functional experiments could be performed only in freshly isolated monocytes and have been done in a limited number of patients followed by the Gaslini Institute. Fresh monocytes were enriched and activated with lipopolysaccharide (LPS) as described $^{11,22}$ in 3 patients carrying the p.Q703K variant, 1 patient with a CINCA phenotype carrying both the p.M406I and p.Q703K, and 7 additional patients with CAPS (Supplementary Table 1, available online at jrheum.org), Six age-matched healthy individuals were used as controls. Cytokines in supernatants were quantified by ELISA assay (R\&D Systems). The Student $t$ test (significance level defined as $p$ values $<0.05$ ) was used for statistical analysis.

\section{RESULTS}

Prevalence of the p.Q703K variant in patients with suspected CAPS and in the normal population. From March 2002 to January 2013, the p.Q703K (p. Q705K, c. 2107C>A) variant was detected in 57/580 white patients (41 children and 16 adults). Fifty-five were heterozygous and 2 were homozygous. The overall allelic frequency was $5 \%$. Twenty-seven patients were exclusively screened for NLRP3, whereas additional genes associated to periodic fevers were analyzed in the remaining 30 patients (52\%; Supplementary Figure 1, available online at jrheum.org).

The clinical features at the moment of genetic analysis were available for 46 patients and are summarized in Supplementary Table 2 (available online at jrheum.org). No positive family history of urticarial skin rash, unexplained fever, or other inflammatory manifestations possibly related to a CAPS-like phenotype was reported in any of the patients. Molecular analysis for Q703K was performed in 1 or 2 parents for 15 patients. In 12 patients, 1 asymptomatic parent carrying the same variant was identified. In the remaining 3 patients, 1 parent only was analyzed.

To estimate the prevalence of the p.Q703K mutation in the general population, 90 healthy individuals were also analyzed. Ten individuals displayed a p.Q703K heterozygous mutation with an overall allelic frequency of $5.5 \%$. Such a

Personal non-commercial use only. The Journal of Rheumatology Copyright @ 2016. All rights reserved 
Table 1. Demographic features of the patients at the time of molecular analysis.

\begin{tabular}{cccc}
\hline Patient $\quad$ Sex & $\begin{array}{c}\text { Age at } \\
\text { Onset, yrs }\end{array}$ & $\begin{array}{c}\text { Age at } \\
\text { Molecular } \\
\text { Analysis, yrs }\end{array}$ & $\begin{array}{c}\text { Mutations in NLRP3 } \\
\text { or in Other Genes }\end{array}$ \\
\hline
\end{tabular}

\begin{tabular}{|c|c|c|c|c|}
\hline S1 & $\mathrm{F}$ & 30 & 41 & Q703K \\
\hline $\mathrm{S} 2$ & $\mathrm{~F}$ & 45 & 60 & Q703K \\
\hline S3 & $\mathrm{F}$ & 22 & 26 & Q703K \\
\hline S4 & $\mathrm{F}$ & 7 & 11 & Q703K \\
\hline S5 & $\mathrm{F}$ & 4 & 21 & Q703K \\
\hline S6 & $\mathrm{M}$ & 17 & 25 & Q703K \\
\hline S7 & $\mathrm{F}$ & 15 & 19 & Q703K \\
\hline S8 & $\mathrm{F}$ & 57 & 58 & Q703K \\
\hline S9 & $\mathrm{F}$ & 28 & 28 & Q703K \\
\hline S10 & $\mathrm{F}$ & 57 & 64 & Q703K \\
\hline S11 & M & 33 & 43 & Q703K \\
\hline $\mathrm{S} 12$ & $\mathrm{~F}$ & 10 & 23 & Q703K \\
\hline S13 & $\mathrm{F}$ & 33 & 38 & Q703K \\
\hline S14 & $\mathrm{M}$ & 15 & 45 & Q703K \\
\hline S15 & $\mathrm{M}$ & 6 & 41 & Q703K \\
\hline S16 & $\mathrm{M}$ & 8 & 8 & Q703K \\
\hline S17 & $\mathrm{F}$ & 1 & 6 & Q703K \\
\hline S18 & $\mathrm{F}$ & 1 & 8 & Q703K \\
\hline S19 & $\mathrm{F}$ & 1 & 7 & Q703K \\
\hline $\mathrm{S} 20$ & M & 1 & 9 & Q703K \\
\hline $\mathrm{S} 21$ & $\mathrm{~F}$ & 3 & 16 & Q703K \\
\hline $\mathrm{S} 22$ & $\mathrm{~F}$ & 4 & 7 & Q703K \\
\hline S23 & $\mathrm{M}$ & 3 & 3 & Q703K \\
\hline S24 & M & 1 & 1 & Q703K \\
\hline S25 & M & 1 & 1 & Q703K \\
\hline S26 & $\mathrm{M}$ & 2 & 4 & Q703K-M406I \\
\hline S27 & $\mathrm{F}$ & 29 & 37 & Q703K \\
\hline $\mathrm{S} 28$ & $\mathrm{~F}$ & 14 & 16 & Q703K \\
\hline S29 & M & 3 & 11 & Q703K \\
\hline S30 & M & 1 & 1 & Q703K \\
\hline S31 & $\mathrm{M}$ & 21 & 23 & Q703K \\
\hline S32 & $\mathrm{F}$ & 1 & 1 & Q703K \\
\hline S33 & M & 5 & 6 & Q703K/V726A $(M E F V)$ \\
\hline S34 & M & 1 & 5 & Q703K \\
\hline S35 & $\mathrm{F}$ & 1 & 2 & Q703K \\
\hline S36 & $\mathrm{F}$ & 1 & 1 & Q703K-D303N + V198M \\
\hline
\end{tabular}

control frequency estimate has been compared with data from the 1000 Genomes project, HapMap, and Exome Aggregation Consortium (ExAC), available in dbSNP (www.ncbi.nlm.nih.gov/SNP) or Ensembl (www.ensembl.org), finding that the " $A$ " allele, associated with the variant protein, was reported with frequencies of $5.1 \%, 5.8 \%$, and $4.1 \%$, respectively. The former 2 frequencies were referred to the European/white population while the latter one was calculated on the basis of a mixed group of 60,706 subjects.

Longterm followup. Complete clinical data at the moment of the molecular analysis and at the last followup were available in 36 patients ( 20 children and 16 adults). The remaining 21 patients were lost to followup by the centers (Supplementary Figure 1, available online at jrheum.org).

The main clinical findings observed in the 36 patients carrying the p.Q703K (p. Q705K, c. 2107C >A) substitution of NLRP 3 with complete followup data are reported in Table 1 and Table 2. The mean age at presentation was 3 years (range 1-14) for pediatric patients and 26.3 years (range 4-57) for adult patients. The mean followup after molecular analysis was 2.5 years (range $0.6-8$ ).

Additional NLRP3 mutations were found in 2 patients: M406I in S26 and D303N + V198M in S36 (Table 1). Additional genes were searched in 18 patients. One patient displayed a monoallelic V726A variant of the $M E F V$ gene (Table 1). The 2 patients who carried additional NLRP3 mutations displayed a clinical phenotype consistent with a diagnosis of CAPS: patient S36 displayed a clinical phenotype characterized by recurrent episodes of fever, urticarial rash, arthralgia, and arthritis associated to elevation of acute-phase reactants consistent with an MWS phenotype, and patient S26, already described ${ }^{12}$, displayed a clear CINCA/NOMID phenotype characterized by persistent inflammation, headache, hearing loss, mental retardation, and typical facies (frontal bossing and midface hypoplasia) without a typical urticarial rash (Table 2).

At the last followup, 13 out of the 34 remaining patients (39\%) were classified by the centers with a diagnosis different from the original suspicion of CAPS. Three patients (S20, S28, and S30) displayed a clinical phenotype characterized by intermittent fever, arthralgia/arthritis, and recurrent urticarial skin that, according to the physicians in charge, was consistent with a systemic-onset juvenile idiopathic arthritis (SoJIA). Five patients (S5, S14, S18, S19, and S23) screened for $N L P R 3$ for recurrent urticarial rash, despite the lack of clear signs of systemic inflammation, were classified as idiopathic urticaria. Four patients (S9, S17, S25, and S33) displayed recurrent fever episodes or chronic inflammation without signs of urticarial rash, receiving a final classification of undifferentiated periodic fevers (Table 2). One patient (S34) was screened because of the presence of an isolated overgrowth of distal phalanges, in the absence of a clear history of persistent or recurrent inflammation and urticarial rash. A final diagnosis of primary hypertrophic osteoarthropathy was pointed out (Table 2).

According to the centers, at the last followup, 21 patients presented an inflammatory phenotype consistent with the original suspicion of a CAPS-like disorder. Nine patients presented a history of chronic or subchronic inflammation with elevation of acute-phase reactants associated with persistent or recurrent urticarial rash and muscle-skeletal manifestations. Twelve patients displayed a clear recurrent disease course with episodes of rash and arthralgia associated with systemic inflammation of variable duration alternating with periods of complete well-being. Six patients $(50 \%)$ with a recurrent disease course presented 1 or 2 episodes/year only. None of the 21 patients displayed complications possibly associated to a severe CAPS such as hearing loss, mental retardation, eye manifestations (e.g., papilledema), or amyloidosis (Table 2). One patient (S24) displayed some

Personal non-commercial use only. The Journal of Rheumatology Copyright @ 2016 . All rights reserved. 

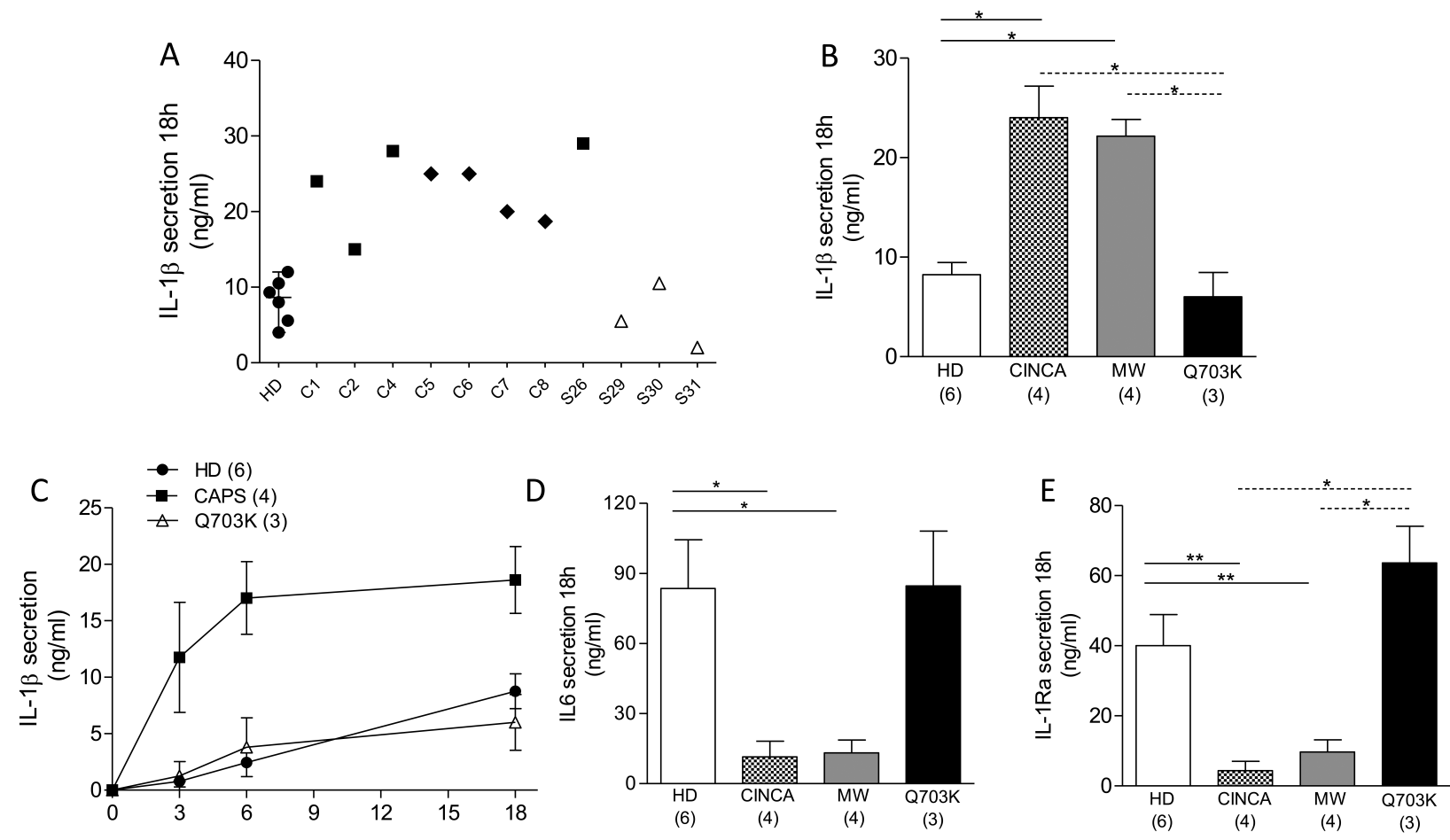

Figure 1. Pattern of cytokine secretion in patients carrying the Q703K variant compared with healthy controls and patients with CAPS. A. Secretion of IL-1 $\beta$ after $18 \mathrm{~h}$ of stimulation with LPS in patients carrying the Q703K variant only (white triangles), Muckle-Wells patients (black rhombus), patients with CINCA (black squares), and age-matched healthy controls (black circles). Patient S26 displayed a CINCA phenotype and was a carrier of either the M406I or Q703K variants. B. Comparison of secretion of IL-1 $\beta$ in the 4 subgroups of patients (*p $<0.05$ at nonparametric test). S26 is included in the CINCA group. C. Kinetics of IL-1 $\beta$ secretion in 4 patients with CAPS (C1, C2, C6, C7), 6 healthy controls (HD), and 3 patients carrying the Q703K variant only. In panels D and E, comparison of secretion of IL-6 and IL-1Ra, respectively, in the 4 subgroups of patients (*p < 0.05 at nonparametric tests, $\left.{ }^{* *} \mathrm{p}<0.01\right)$. S26 is included in the CINCA group. CAPS: cryopyrin-associated periodic syndrome; IL-1: interleukin 1; LPS: lipopolysaccharide; CINCA: chronic infantile neurologic, cutaneous, articular syndrome; IL-1Ra: IL-1 receptor antagonist; MW: Muckle-Wells.

dysmorphic features (gross face and skeletal dysplasia) and slight mental retardation, which according to the expert physician in charge, were not compatible with a CINCA/NOMID phenotype.

The therapeutic strategy used in the 21 patients carrying the Q703K NLRP3 mutation with CAPS-like manifestations is reported in Supplementary Figure 2 (available online at jrheum.org). Steroids were used in $16 / 21$ patients (12 on-demand vs 4 continuous treatment), often in association with nonsteroidal antiinflammatory drugs. In 7 out of 16 patients (44\%), this strategy led to a complete control of clinical manifestations, especially in those patients presenting a recurrent or episodic disease course. Anti-IL-1 treatment (anakinra) was attempted in 8 patients (38\%) displaying a chronic or subchronic disease course, requiring continuous steroid treatment or a frequent use of steroid on demand. A complete normalization of the clinical manifestations and laboratory variables was observed in $5 / 8$ patients $(62 \%$, complete responders), whereas $3 / 8$ patients experienced a partial or absent response, with withdrawal of the treatment after some weeks or months (nonresponders). None of the 5 complete responder patients was to date investigated for a possible somatic mosaicism of $N L R P 3$ or for mutations in other exons of the gene. Other treatments were used with incomplete or no response, such as anti-TNF (3 patients), colchicine (2 patients), and various combinations of diseasemodifying antirheumatic drugs (3 patients; Table 2; Supplementary Figure 2, available online at jrheum.org).

Pattern of IL-1 $\beta$ secretion in patients with $Q 703 K$ compared with healthy controls and patients with CAPS. Fresh monocytes from 3 patients carrying the p.Q703K (p. Q705K, c. $2107 \mathrm{C}>\mathrm{A})$ variant $(\mathrm{S} 29, \mathrm{~S} 30, \mathrm{~S} 31)$ and 1 patient with a CINCA phenotype carrying both the p.M406I and p.Q703K variants (S26) were analyzed for the IL-1 secretion, and compared with monocytes from 7 patients with CAPS (3 CINCA and 4 MWS) carrying variants surely pathogenic and from 6 age-matched healthy controls, as reported ${ }^{11,22}$.

The genotype/phenotype correlation, ongoing disease activity, and treatment at the moment of the study of the patients analyzed are reported in Supplementary Table 1 (available online at jrheum.org).

As shown in Figure 1A, the level of secretion after $18 \mathrm{~h}$ of stimulation with LPS in monocytes from the 3 patients carrying uniquely the p.Q703K variant (S29, S30, S31) was

Personal non-commercial use only. The Journal of Rheumatology Copyright @ 2016 . All rights reserved. 


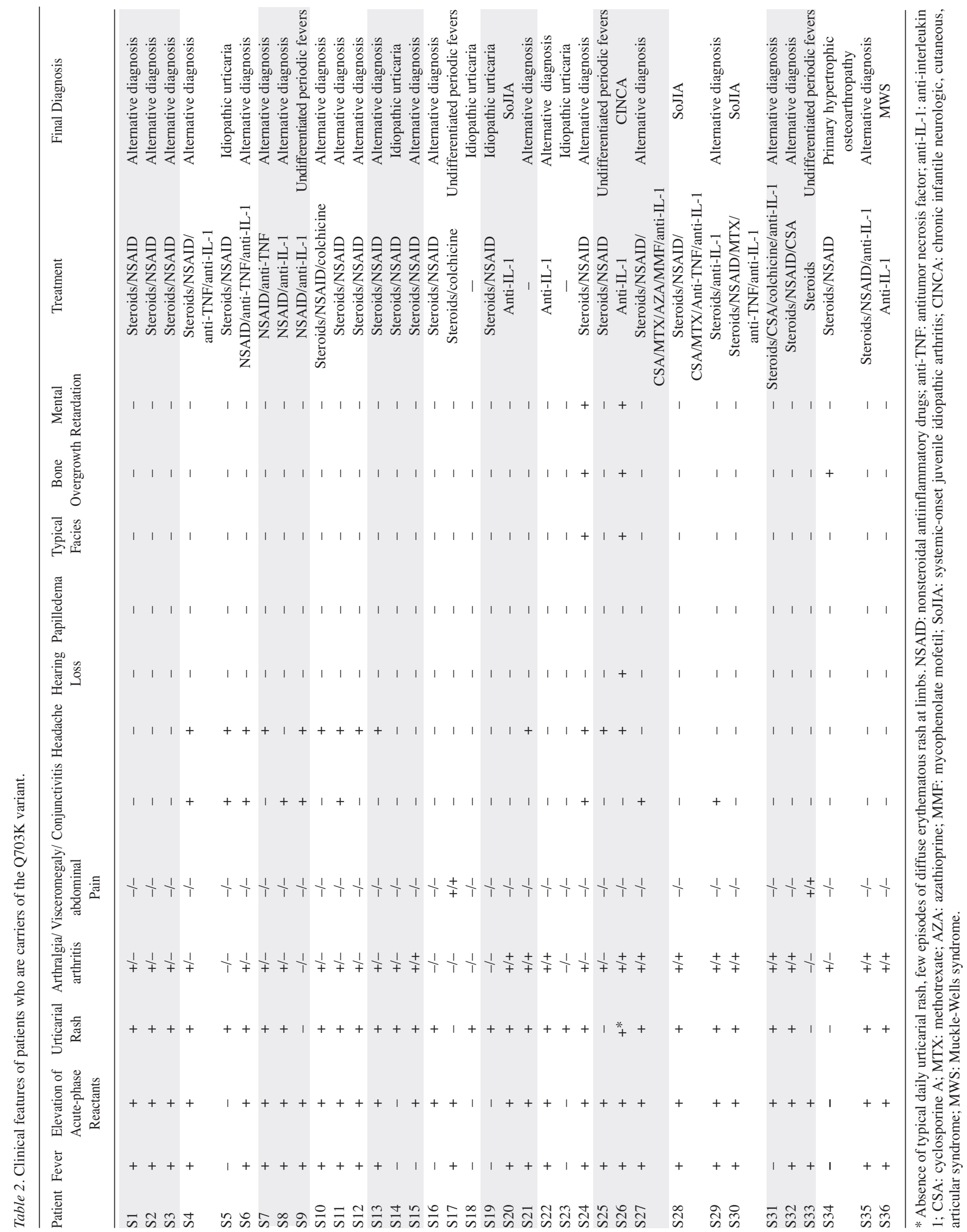

Personal non-commercial use only. The Journal of Rheumatology Copyright @ 2016. All rights reserved. 
comparable to that observed in healthy controls and much less than that found in monocytes from patients with MWS and CINCA. Conversely, patient S26, carrying an additional NLRP 3 mutation and displaying a severe CINCA phenotype, displayed the typical IL-1 $\beta$ over-secretion observed in patients with CAPS (Figure 1A and Figure 1B). In previous studies, we have shown that monocytes from patients with CAPS displayed peculiar kinetics of IL-1 secretion that reached the peak at 3-6 $\mathrm{h}$ after LPS stimulation, well before monocytes from healthy subjects ${ }^{23}$. Conversely, patients carrying the $\mathrm{p} . \mathrm{Q} 703 \mathrm{~K}$ variant show a kinetics comparable to those observed in healthy controls (Figure 1C). Monocytes from patients with CAPS displayed impaired secretion of second-wave cytokines such as IL-6 and IL-1 receptor antagonist (IL-1Ra) independently of disease activity and ongoing treatment ${ }^{22}$. This is due to the insurgence of oxidative stress in CAPS monocytes following Toll-like receptor stimulation. In fact, a common cell response to oxidative stress is the block of protein synthesis, which occurs in stressed CAPS monocytes with consequent impaired translation of IL-1Ra and IL-6 mRNA ${ }^{22,24}$. Unlike monocytes from patients with CAPS, monocytes from patients carrying uniquely the p.Q703K variant displayed a normal secretion of these 2 cytokines (Figure 1D and Figure 1E, respectively).

\section{DISCUSSION}

In our current study, we analyze the clinical phenotype and longterm followup of a selected population of patients screened for NLRP3 for a suspected CAPS in 2 national referral centers during the last 10 years and found to be positive for the p.Q703K (p. Q705K, c. 2107C>A) variant. Despite the evident bias of selection toward patients with a possible CAPS-like phenotype, the results of our study add further evidence to clarify the poor clinical significance of this variant. These conclusions are driven by the high proportion of patients receiving a final alternative diagnosis and by the observation of the same prevalence of the p.Q703K variant in this selected group of patients when compared with the healthy population. Moreover, most of the patients carrying this variant displayed a very mild phenotype with the absence of the most typical CAPS-associated manifestations (hearing loss, neurological involvement, bone dysplasia). In contrast with "classical" CAPS, a low percentage of patients carrying the Q703K variant required an anti-IL-1 treatment, with a relatively high proportion of poor responders. The p.Q703K variant of the NLRP3 gene has been considered for a long time an unremarkable, likely neutral polymorphism ${ }^{4,17}$. This policy was challenged by the description of a patient carrying the p.Q703K showing a clinical phenotype consistent with CAPS with a good response to anti-IL- 1 treatment ${ }^{18}$. The patient's monocytes showed an overactivation of caspase 1 and higher amounts of secreted active IL- $1 \beta$ after LPS stimulation compared with 5 healthy controls ${ }^{18}$. The same group performed an in vitro study transfecting the p.Q703K into the human monocytic cell line THP- $1^{19}$. After stimulation, THP-1 cells transduced with NLRP3-Q703K secreted higher levels of IL-1 $\beta$ compared with NLRP3-WT, but much less compared with the NLRP3-R260W variant, a proven pathogenic NLRP3 variant usually associated to a mild form of CAPS (FCAS and MWS ${ }^{19}$.

After this report ${ }^{19}$, a note added to the guidelines for the genetic diagnosis of hereditary recurrent fevers pointed out the possibility that this variant could be considered in the group of variants of uncertain significance and therefore recommended that it be reported to the clinicians ${ }^{17}$.

In our present study, the unique criterion for the analysis of the $N L R P 3$ gene was the request from the referral center. In more than $50 \%$ of patients, a gene other than NLRP3 was requested for screening, likely because of the presence of an undefined phenotype. In addition to patients identified by experienced physicians, there were a number of patients directly referred by local hospitals with limited experience in autoinflammatory diseases. This explains the variability of the clinical phenotypes reported at the time of molecular analysis and also the rather relevant number of patients presenting features not consistent with a possible CAPS. The absent elevation of acute-phase reactants should be considered a criterion of exclusion for the request of the molecular analysis for NLRP3.

Therefore, we decided to focus the analysis of the actual clinical effect of the p.Q703K variant only on those patients for whom followup data were available. According to the evaluations by the centers, in $38 \%$ of the patients the final diagnosis at the last followup was not consistent with the original suspicion of a CAPS-like phenotype. After followup, 21 patients presented some clinical features still consistent with the original suspicion of a CAPS-like disorder, generally with a very mild clinical course. Most of the patients presented a recurrent disease course with few episodes during the year. None of these patients developed clinical features classically associated to a severe CAPS phenotype (skeletal and neurological involvement, hearing loss, papilledema). These data are in contrast with a recent report showing a possible correlation between the presence of NLRP3 low penetrance mutations and a variable spectrum of neurological manifestations ${ }^{25}$. Moreover, in contrast with the common finding usually observed in FCAS and MWS, no clear positive family history was observed. These findings reduce the actual pathogenic significance of the p.Q703K NLRP3 variant and can be explained by the relatively low specificity of the clinical manifestations presented by the patients at the moment of molecular analysis, essentially consisting of systemic inflammation associated with some urticarial rash of variable duration and severity. It should be noted that the good response to anti-IL-1 treatment is not strictly limited to CAPS but is also observed in a number of other genetic and multifactorial disorders, including patients with a CAPS-like

Personal non-commercial use only. The Journal of Rheumatology Copyright @ 2016. All rights reserved. 
phenotype but negative for mutations of the NLRP3 gene g, $^{27}$. On the other hand, we cannot exclude, in the few patients with a more severe CAPS-like phenotype presenting a complete response to anti-IL-1 blockade, the presence of either NLRP3 germ line mutations in unscreened gene regions or NLRP3 somatic mutations, these latter having been detected in CAPS and recognized as causative of even severe disease forms ${ }^{8}$.

The limited pathogenic effect of the p.Q703K variant is further supported by our experimental approach on patients' primary cells. In the last few years, we characterized in detail the peculiar pattern of activation and secretion of IL- $1 \beta$ in patients with CAPS carrying variants of certain pathogenic effect with different degrees of disease severity $11,22,23$. Because of a severe alteration in the redox balance, NLRP3-mutated monocytes display a fast activation and secretion of IL- $1 \beta$, followed by an exhaustion of their capacity to further produce other proteins and cytokines, such as IL-6 and IL-1Ra $\mathrm{R}^{22,23}$. This immunological phenotype is specific for CAPS and is not observed in inflammatory conditions, such as SoJIA ${ }^{23}$ and $\mathrm{FMF}^{24}$. In our study, we were not able to replicate the results obtained by Verma, et al in a single patient and in their in vitro study ${ }^{18,19}$. Independently from their disease activity and final diagnosis, the pattern of cytokine secretion of patients carrying the p.Q703K variant was similar to those observed in healthy individuals and not comparable to those of patients with CAPS carrying sure pathogenic variants. This observation is in line with a recent study by Rieber, et al showing a clear difference in the production of IL-1 $\beta$, IL-18, and caspase 1 in patients with low penetrance mutations compared to patients carrying pathogenic variants ${ }^{28}$.

The relatively small number of patients and healthy controls analyzed in our present study does not allow us to definitively establish or refute a causal relationship from the genetic point of view. Nonetheless, because similar Q703K variant frequencies (range 4.1-5.8\%) were reported in a very large number of subjects in the 1000 Genomes project, HapMap, and ExAC, we can reasonably conclude that there is no difference between patients and healthy controls regarding the presence of the variant "A" allele. In any case, the evidence we report suggests that the $N L R P 3$ p.Q703K variant should be considered a polymorphism without an evident functional and clinical effect. This is new evidence against the need to report this variant after genetic analysis, as originally suggested by the guidelines proposed by Shinar, et $a l^{17}$.

\section{ONLINE SUPPLEMENT}

Supplementary data for this article are available online at jrheum.org.

\section{REFERENCES}

1. Hoffman HM, Mueller JL, Broide DH, Wanderer AA, Kolodner RD. Mutation of a new gene encoding a putative pyrin-like protein causes familial cold autoinflammatory syndrome and Muckle-Wells syndrome. Nat Genet 2001;29:301-5.
2. Kile RL, Rusk HA. A case of cold urticaria with an unusual family history. JAMA 1940;114:1067-8.

3. Neven B, Callebaut I, Prieur AM, Feldmann J, Bodemer C, Lepore $\mathrm{L}$, et al. Molecular basis of the spectral expression of CIAS1 mutations associated with phagocytic cell-mediated autoinflammatory disorders CINCA/NOMID, MWS, and FCU. Blood 2004;103:2809-15.

4. Aksentijevich I, D Putnam C, Remmers EF, Mueller JL, Le J, Kolodner RD, et al. The clinical continuum of cryopyrinopathies: novel CIAS1 mutations in North American patients and a new cryopyrin model. Arthritis Rheum 2007;56:1273-85.

5. Muckle TJ, Wells M. Urticaria, deafness, and amyloidosis: a new heredo-familial syndrome. Q J Med 1962;31:235-48.

6. Prieur AM, Griscelli C. Arthropathy with rash, chronic meningitis, eye lesions, and mental retardation. J Pediatr 1981;99:79-83.

7. Milhavet F, Cuisset L, Hoffman HM, Slim R, El-Shanti H, Aksentijevich I, et al. The infevers autoinflammatory mutation online registry: update with new genes and functions. Hum Mutat 2008:29:803-8.

8. Tanaka N, Izawa K, Saito MK, Sakuma M, Oshima K, Ohara O, et al. High incidence of NLRP3 somatic mosaicism in patients with chronic infantile neurologic, cutaneous, articular syndrome: results of an International Multicenter Collaborative Study. Arthritis Rheum 2011;63:3625-32.

9. Nakagawa K, Gonzalez-Roca E, Souto A, Kawai T, Umebayashi H, Campistol JM, et al. Somatic NLRP3 mosaicism in Muckle-Wells syndrome. A genetic mechanism shared by different phenotypes of cryopyrin-associated periodic syndromes. Ann Rheum Dis 2015;74:603-10.

10. Agostini L, Martinon F, Burns K, McDermott MF, Hawkins PN, Tschopp J. NALP3 forms an IL-1beta-processing inflammasome with increased activity in Muckle-Wells autoinflammatory disorder. Immunity 2004;20:319-25.

11. Gattorno M, Tassi S, Carta S, Delfino L, Ferlito F, Pelagatti MA, et al. Pattern of interleukin-1beta secretion in response to lipopolysaccharide and ATP before and after interleukin-1 blockade in patients with CIAS1 mutations. Arthritis Rheum 2007; 56:3138-48.

12. Lepore L, Paloni G, Caorsi R, Alessio M, Rigante D, Ruperto N, et al. Follow-up and quality of life of patients with cryopyrin-associated periodic syndromes treated with anakinra. J Pediatr 2010;157:310-5.

13. Lachmann HJ, Kone-Paut I, Kuemmerle-Deschner JB, Leslie KS, Hachulla E, Quartier P, et al; Canakinumab in CAPS Study Group. Use of canakinumab in the cryopyrin-associated periodic syndrome. N Engl J Med 2009;360:2416-25.

14. Hoffman HM. Rilonacept for the treatment of cryopyrin-associated periodic syndromes (CAPS). Expert Opin Biol Ther 2009;9:519-31.

15. Levy R, Gérard L, Kuemmerle-Deschner J, Lachmann HJ, Koné-Paut I, Cantarini L, et al. Phenotypic and genotypic characteristics of cryopyrin-associated periodic syndrome: a series of 136 patients from the Eurofever Registry. Ann Rheum Dis 2015;74:2043-9.

16. Rowczenio DM, Trojer H, Russell T, Baginska A, Lane T, Stewart NM, et al. Clinical characteristics in subjects with NLRP3 V198M diagnosed at a single UK center and a review of the literature. Arthritis Res Ther 2013;15:R30.

17. Shinar Y, Obici L, Aksentijevich I, Bennetts B, Austrup F, Ceccherini I, et al. Guidelines for the genetic diagnosis of hereditary recurrent fevers. Ann Rheum Dis 2012;71:1599-605.

18. Verma D, Lerm M, Blomgran Julinder R, Eriksson P, Söderkvist P, Särndahl E. Gene polymorphisms in the NALP3 inflammasome are associated with interleukin-1 production and severe inflammation: relation to common inflammatory diseases? Arthritis Rheum 2008;58:888-94.

Personal non-commercial use only. The Journal of Rheumatology Copyright $\subset$ 2016. All rights reserved. 
19. Verma D, Särndahl E, Andersson H, Eriksson P, Fredrikson M, Jönsson JI, et al. The Q705K polymorphism in NLRP3 is a gain-of-function alteration leading to excessive interleukin- $1 \beta$ and IL-18 production. PLoS One 2012;7:e34977.

20. Vitale A, Lucherini OM, Galeazzi M, Frediani B, Cantarini L. Long-term clinical course of patients carrying the Q703K mutation in the NLRP3 gene: a case series. Clin Exp Rheumatol 2012;30:943-6.

21. Caroli F, Pontillo A, D’Osualdo A, Travan L, Ceccherini I, Crovella $\mathrm{S}$, et al. Clinical and genetic characterization of Italian patients affected by CINCA syndrome. Rheumatology 2007;46:473-8.

22. Carta S, Tassi S, Delfino L, Omenetti A, Raffa S, Torrisi MR, et al. Deficient production of IL-1 receptor antagonist and IL-6 coupled to oxidative stress in cryopyrin-associated periodic syndrome monocytes. Ann Rheum Dis 2012;71:1577-81.

23. Tassi S, Carta S, Delfino L, Caorsi R, Martini A, Gattorno M, et al. Altered redox state of monocytes from cryopyrin-associated periodic syndromes causes accelerated IL-1beta secretion. Proc Natl Acad Sci U S A 2010;107:9789-94.
24. Omenetti A, Carta S, Delfino L, Martini A, Gattorno M, Rubartelli A. Increased NLRP3-dependent interleukin $1 \beta$ secretion in patients with familial Mediterranean fever: correlation with MEFV genotype. Ann Rheum Dis 2014;73:462-9.

25. Schuh E, Lohse P, Ertl-Wagner B, Witt M, Krumbholz M, Frankenberger M, et al. Expanding spectrum of neurologic manifestations in patients with NLRP3 low-penetrance mutations. Neurol Neuroimmunol Neuroinflamm 2015;2:e109.

26. Gattorno M, Martini A. Beyond the NLRP3 inflammasome: autoinflammatory diseases reach adolescence. Arthritis Rheum 2013;65:1137-47.

27. Caorsi R, Federici S, Gattorno M. Biologic drugs in autoinflammatory syndromes. Autoimmun Rev 2012;12:81-6.

28. Rieber N, Gavrilov A, Hofer L, Singh A, Öz H, Endres T, et al. A functional inflammasome activation assay differentiates patients with pathogenic NLRP3 mutations and symptomatic patients with low penetrance variants. Clin Immunol 2015;157:56-64. 


\section{Correction}

Clinical Characteristics of Patients Carrying the Q703K Variant of the NLRP3 Gene: A 10-year Multicentric National Study

Aldo Naselli, Federica Penco, Luca Cantarini, Antonella Insalaco, Mariolina Alessio, Alberto Tommasini, Cristina Maggio, Laura Obici, Romina Gallizzi, Marco Cimmino, Sara Signa, Orso Maria Lucherini, Sonia Carta, Francesco Caroli, Alberto Martini, Anna Rubartelli, Isabella Ceccherini, and Marco Gattorno

J Rheumatol 2016;43:1093-100; doi: 10.3899/jrheum.150962

The correct last name of the coauthor R. Gallizi is Romina Gallizzi.

doi: 10.3899/jrheum.150962.C1 